(Aus der Frauenklinik von Geheimrath Prof. Dr. L. und Dr. Th. Landau, Berlin.)

\title{
Das klinische Verhalten des Adenomyoma uteri.
}

\author{
Von \\ Dr. D. Grünbaum, \\ Frauenarzt in Nürnberg; өhem. I. Assistent der Klinik. \\ (Mit 1 Textfigur.)
}

Dem grundlegenden Werke v. Recklinghausen's über die Adenomyome und Cystadenome des Uterus gliederte W. A. Freund kurze klinische Notizen über die voluminösen Adenomyome des Uterus an, in welchen er den Nachweis zu erbringen versucht, dass die pathologisch-anatomisch von den Uterusmyomen leicht zu trennenden Adenomyome auch klinisch von denselben verschieden sind und ein gut erkennbares Krankheitsbild hervorrufen.

Anamnestisch ergebe sich, dass die an Adenomyom Erkrankten meist eine schwächliche Kindheit hinter sich hätten und die Menstruation sich später als gewöhnlich einstelle. Sehr häufig sei die Menstruation stark und mit grossen Schmerzen verbunden; unregelmässige Blutungen seien die Klagen der Patientinnen. Das Allgemeinbefinden sei beträchtlich gestört, und das längere Bestehen der Geschwulst führe zur vollständigen Arbeitsunfähigkeit.

Objectiv finden sich neben Zeichen von allgemeiner Hypoplasie und Infantilismus ausser den tumorartigen Hervorragungen an der Hinterseite der Gebärmutter Residuen abgelaufener Pelveoperitonitis mit Fixation der Beckenorgane. Die Wachsthumsrichtung der Adenomyome verlaufe oft in charakteristischer Weise vom Tubenwinkel nach abwärts an die Cervix hin.

Freund's Ausführungen blieben nicht lange unwidersprochen. L. Pick glaubte durch Vergleichung der pathologisch-anatomischen 
Befunde v. Recklinghausen's mit den von Freund klinisch analysirten Tumoren nachweisen za können, dass das Krankheitsbild nicht in allen Punkten stimmt, und er zweifelt an der Möglichkeit einer exacten Diagnose des Adenomyoms.

L. Landau widmete in seinen anatomischen und klinischen Beiträgen zur Lehre der Myome auch dem klinischen Verhalten des Adenomyoms einen eigenen Abschnitt; an der Hand des damals noch spärlichen Materials kommt L. Landau im Gegensatz zu W. A. Freund zu dem Schluss, dass die Symptomatologie der Adenomyome, sofern sie überhaupt klinische Symptome machen, sich von der der Myome gemeinhin nicht unterscheidet. Nur in Bezug auf die Therapie ist die Trennung der Myome von den Adenomyomen gerechtfertigt; in diesem Punkt stimmt L. Landau mit W. A. Freund überein, dass für die grossen Adenomyome nur die Totalexstirpation in Frage kommt, da das Adenomyom durch sein inniges Verbundensein mit dem Uterus, durch seinen infiltrativen Charakter sich nicht in toto so ausschälen lässt, wie das Kugelmyom. Bei den neben dem Uterus gelegenen paroophoralen Tumoren ist eine Conservirung des Uterus selbst möglich. Bezüglich der Prognose weist L. Landau besonders darauf hin, dass das Adenomyom insofern für die Trägerin eine günstige Aussicht bietet, als regressive Metamorphosen, wie Nekrose, Vereiterungen, Gangrän, die beim Myom lebensbedrohliche Erscheinungen machen können, beim Adenomyom nicht beobachtet werden. Andererseits aber gebe es beim Adonomyom wegen seiner diffusen Verankerung mit dem Mutterboden keine Expulsion des Tumors mit spontaner Heilung.

Auf Grund einer eingehenden Betrachtung des vorliegenden kasuistischen Materials von 100 Fällen kommen Kudoh und Polano zu einer vollen Bestätigung der Ansichten L. Landau's. Eine scharfe klinische Abgrenzung des Krankheitsbildes im Sinne Freund's sei in der überwiegenden Mehrzahl der Fälle unmöglich, da alle charakteristischen Symptome bei typischen Adenomyomfällen fehlen und beim einfachen Fibromyom vorhanden sein können. Auffallend sei das häufige Vorkommen von Adhäsionen und Adnexentzündungen beim Adenomyom, und das Fehlen von Degenerationsprocessen am Tumor selbst 1 ).

1) Gegen die Statistik von Kudoh ist einzuwenden, dass die Auswahl der Fälle aus der Literatur nicht ganz sorgfältig vorgenommen wurde; einige 
Ein eifriger Vorkämpfer für die Freund'schen Ansebaungen ist Funk, der in der That über 2 Fälle berichten konnte, in denen die Diagnose auf Adenomyom vor der Operation gestellt wurde und zwar auf Grund der Wachsthumsrichtung der Tumoren vom Tubenwinkel nach abwärts und der Schwerbeweglichkeit des Uterus, welche auf Adhaesionen schliessen liess. Nach Funk fallen für die Diagnose hauptsächlich diese beiden Punkte, die Wachsthumsrichtung des Tumors und die Combination mit Pelveoperitonitis und Adnextumoren in's Gewicht. Das Adenomyom führe schon bei viel geringerer Grösse Adhaesionen herbei als das Kugelmyom. Funk wendet sich in seinen Ausführungen vor allem gegen L. Pick, der die Möglichkeit einer präcisen Diagnosenstellung des Adenomyoms in Frage stellte.

Endlich brachte v. Rosthorn 2 Fälle von Adenomyom zur Veröffentlichung, die besonders in den anamnestischen Daten mit den von W. A. Freund angegebenen übereinstimmen. Die Diagnose wurde in beiden. Fällen nicht gestellt. v. Rosthorn resümirt, dass es viele Fälle von Adenomyom giebt, in denen das klinische Bild kein bestimmtes ist; es würde sich auch in $\mathrm{Zn}$ kunft nur um eine Wahrscheinlichkeitsdiagnose handeln können.

Es ist aus den bisherigen Arbeiten, von denen ich nur die wichtigsten anführte, ersichtlich, dass die Frage nach dem klinischen Verhalten des Adenomyoma uteri noch eine strittige ist, ${ }^{1}$ ) und ich habe mir deshalb die Aufgabe gestellt, das Adenomyommaterial der Landau'schen Klinik nach klinischen Gesichtspunkten zu sichten, um einen Beitrag zu der Frage zu liefern, ob das Adenomyoma uteri eine scharfe, klinische Abgrenzung von dem Fibromyom zulässt. 20 klinisch gut beobachtete Fälle standen mir zur Verfügung, deren Daten in nachfolgender Tabelle zusammengestellt sind.

Fälle sind in seiner Zusammenstellung doppelt geführt, einige Fälle, die als Adenomyomata uteri figuriren, sind gar keine Adenomyome. Auch bedarf die Statistik einer Ergänzung der in der Literatur niedergelegten Fälle von carcinomàtöser und tuberculöser Degeneration des Adenomyoms.

1) In der neuen Auflage des Handbuches der Gynäkologie von Veit wird die klinische Seite des Adenomyoms gar nicht erörtert. 


\begin{tabular}{|c|c|c|c|c|c|}
\hline 吕 & $\begin{array}{l}\text { Journ. } \\
\text { Nr. }\end{array}$ & & $\begin{array}{l}\text { ?para } \\
\text { ? Abort }\end{array}$ & & A I a $\mathrm{mn}$ e se \\
\hline $1+$ & 2677 & verh. & $\begin{array}{c}0 \text { para } \\
0 \text { Abort }\end{array}$ & 41 & $\begin{array}{l}\text { Starke, unregelmässige Menses mit krampfartigen } \\
\text { Unterleibskschmerzen. Sehr blasse, elend aus- } \\
\text { sehende Frau }\end{array}$ \\
\hline $2+$ & 5356 & verh. & $\begin{array}{c}0 \text { para } \\
0 \text { Abort }\end{array}$ & 38 & $\begin{array}{l}\text { Kräftig gebarte, aber sehr anämische Frau, seit einigen } \\
\text { Jahren profuse Blutungen }\end{array}$ \\
\hline $3 \uparrow$ & 6003 & verh. & $\begin{array}{l}1 \text { para } \\
0 \text { Abort }\end{array}$ & 43 & $\begin{array}{l}\text { Meno-Metrorrhagien; gracile, schlecht genährte } \\
\text { Frau }\end{array}$ \\
\hline $4 \uparrow$ & 6069 & verh. & 1 para & 45 & $\begin{array}{l}\text { Abundante Metrorrhagien; kräftig gebaute, aber sehr } \\
\text { anämische Frau }\end{array}$ \\
\hline 5 & 6089 & verh. & 3 para & 47 & $\begin{array}{l}\text { Kräftige, gut genährte Frau; Drängungsbeschwerden } \\
\text { nach unten }\end{array}$ \\
\hline 6 & 6329 & verh. & $\begin{array}{c}1 p \text { ara } \\
0 \text { Abort }\end{array}$ & 34 & $\begin{array}{l}\text { Mittelgrosse, mässig gut genährte Frau; Menses ein- } \\
\text { tägig, sehr schmerzhaft, alle } 4 \text { Wochen, seit } 7 \text { Jahren } \\
\text { starke Schmerzen im Kreuz u. in der rechten Seite }\end{array}$ \\
\hline 7 & 6146 & verh. & $\begin{array}{l}0 \text { para } \\
0 \text { Abort }\end{array}$ & 35 & $\begin{array}{l}\text { Mittelgrosse, gut genährte Frau; postmenstruelle, starke, } \\
8 \text { Tage dauernde Sehmerzen, Menses mit } 14 \text { Jahren } \\
\text { regelm. } 3 \text { Tage dauernd }\end{array}$ \\
\hline 8 & 9796 & ledig & $\begin{array}{c}0 \text { para } \\
1 \text { Abort }\end{array}$ & 46 & $\begin{array}{l}\text { Seit } 4 \text { Jahren unregelmässige } 14 \text { Tage lang dauernde } \\
\text { Blutungen }\end{array}$ \\
\hline 9 & 10274 & verh. & $\begin{array}{c}0 \text { para } \\
0 \text { Abort }\end{array}$ & 32 & $\begin{array}{l}\text { Menses sehr unregelmässig alle } 6-8 \text { Wochen mit starken } \\
\text { Schmerzen; früher } 2 \text { mal Abrasio. Starker Ausfluss. } \\
\text { Gracile Person in herabgesetztem Ernährungszustand }\end{array}$ \\
\hline 10 & 10526 & verh. & $\begin{array}{c}3 \text { para } \\
0 \text { Abort }\end{array}$ & 40 & $\begin{array}{l}\text { Erste Menses mit } 13 \text { Jahren; seit } 2 \text { Jahren unregel- } \\
\text { mässige Blutungen, seit Monaten trotz Abrasio sehr } \\
\text { starke anhaltende Blutuagen. Kräftige, grosse Frau }\end{array}$ \\
\hline 11 & 10710 & verh. & $\begin{array}{l}0 \text { para } \\
\text { OAbort }\end{array}$ & 43 & Unregelmässige Blutungen \\
\hline 12 & 10770 & verh. & $\begin{array}{l}1 \text { para } \\
0 \text { Abort }\end{array}$ & 51 & $\begin{array}{l}\text { Menses bis vor } 4 \text { Jahren regelmässig; seit dieser Zeit } \\
\text { sehr starke 3-4 Tage dauernde Periode. Schwäch- } \\
\text { liche Frau in schlechtem Ernährungszustand }\end{array}$ \\
\hline 13 & 11313 & verh. & $\begin{array}{c}0 \text { para } \\
\text { 0Abort }\end{array}$ & 4.5 & $\begin{array}{l}\text { Menses } 6-7 \text { Tage dauernd, sehwach, ohne Schmerzen. } \\
\text { Seit } 5 \text { Jahren Blasenbeschwerden, die sich bis } \\
\text { zur Urinverhaltung steigerten. Mittelgrosse Frau in } \\
\text { mässigem Ernährungszustand }\end{array}$ \\
\hline 14 & 11667 & ledig & $\begin{array}{c}0 \text { para } \\
0 \text { Abort }\end{array}$ & 35 & $\begin{array}{l}\text { Menses mit } 15 \text { Jahren zum ersten Male, alle } 3 \text { Wochen } \\
8 \text { Tage dauernd; vor } 3 \text { Jahren Laparotomie wegen } \\
\text { rechtsseit. Tuboovarialcyste; jetzt sehrstarke Schmer- } \\
\text { zen bei der Periode. Kräftige, grosse Person }\end{array}$ \\
\hline 15 & 11897 & verh. & $\begin{array}{c}0 \text { para } \\
0 \text { Abort }\end{array}$ & 50 & $\begin{array}{l}\text { Menses zum ersten Male mit } 13 \text { Jahren; in den letaten } \\
2 \text { Jahren unregelmässig, Blasenbeschwerden. } \\
\text { Mittelgrosse, sehr kräftige Frau }\end{array}$ \\
\hline 16 & 11984 & verh. & $\begin{array}{l}0 \text { para } \\
0 \text { Abort }\end{array}$ & 44 & $\begin{array}{l}\text { Menses zum ersten Male mit } 15 \text { Jahren, seit } 1 / 2 \text { Jahre } \\
\text { unregelm. Blutungen; die Menses dauern 10-14 } \\
\text { Tage und wiederholen sich alle 2-3 Woehen. Beim } \\
\text { Husten unwillkürlicher Urinabgang }\end{array}$ \\
\hline
\end{tabular}

Von den mit $\dagger$ bezeichneten Fällen findet sich ausführliche Krankengeschichten in 


\begin{tabular}{|c|c|c|}
\hline Operation & $B$ ef $u n d$ & \\
\hline $\begin{array}{l}\text { Abdomin. Radicaloperation. } \\
\quad \text { 6. 5. } 1893\end{array}$ & $\begin{array}{l}\text { Adenomyoma paraovorale dorsi uteri. Pyo- } \\
\text { salpinx. Multiple Fibroide. Sehr viele } \\
\text { Adbäsionen }\end{array}$ & $\begin{array}{l}\text { Geheilt entlassen. } \\
\text { 8. 6. } 1893\end{array}$ \\
\hline $\begin{array}{l}\text { Abdomin. Radicaloperation. } \\
\text { 16.12. } 1897\end{array}$ & $\begin{array}{l}\text { Adenomyoma corporis uteri. Tuboovarial- } \\
\text { cyste links. Geringe Adhäsionen }\end{array}$ & $\begin{array}{l}\text { Geheilt entlassen. } \\
\text { 16. 1. } 1896\end{array}$ \\
\hline $\begin{array}{l}\text { Vaginale } \text { Radicaloperation. } \\
\text { 20.12. } 1898\end{array}$ & $\begin{array}{l}\text { Adenomyoma fundi uteri. Geringe Ad- } \\
\text { häsionen }\end{array}$ & $\begin{array}{l}\text { Geheilt entlassen. } \\
\text { 9. 1. } 1899\end{array}$ \\
\hline $\begin{array}{l}\text { Vaginale Radicaloperation. } \\
\text { 31. 1. } 1899\end{array}$ & $\begin{array}{l}\text { Adenomyoma fundi uteri, Salpingitis dup- } \\
\text { lex. Degeneratio cystica ovarii sinistri. } \\
\text { Starke Adhäsionsbildung }\end{array}$ & $\begin{array}{l}\text { Geheilt entlassen. } \\
\text { 18. 2. } 1899\end{array}$ \\
\hline $\begin{array}{l}\text { Vaginale Radicaloperation. } \\
\text { 7. 2. } 1899\end{array}$ & $\begin{array}{l}\text { Hyperplasia uteri (corporis et cervicis) } \\
\text { praecipue labiianterioris portionis. Kugel- } \\
\text { myom; multiple subseröse Adenomyome; } \\
\text { Atrophia ovariorum. Keine Adhäsionen }\end{array}$ & $\begin{array}{l}\text { Geheilt entlassen. } \\
\text { 25. 2. } 1899\end{array}$ \\
\hline $\begin{array}{l}\text { Vaginale Radicaloperation. } \\
\text { 8. 7. } 1899\end{array}$ & $\begin{array}{l}\text { Hyperplasia uteri. Adenomyoma fundi uteri, } \\
\text { Pachysalpingitis duplex. Pachypelveo- } \\
\text { peritonitis adhaesiva. Degeneratio cy- } \\
\text { stica ovarii dextri }\end{array}$ & $\begin{array}{l}\text { Geheilt entlassen. } \\
\text { 23. 7. } 1899\end{array}$ \\
\hline $\begin{array}{l}\text { Vaginale Radicaloperation. } \\
\text { 13. 3. } 1899\end{array}$ & $\begin{array}{l}\text { Adenomyoma fundi uteri. Geringe Ad- } \\
\text { häsionen }\end{array}$ & $\begin{array}{l}\text { Geheilt entlassen. } \\
\text { 29. 3. } 1899\end{array}$ \\
\hline $\begin{array}{l}\text { Abdominelle Totalexstirpa- } \\
\text { tion. 7. 6. } 1904\end{array}$ & $\begin{array}{l}\text { Adenomyoma corporis uteri, Hydrosalpinx } \\
\text { duplex. Follikelcyste des linken Ovari- } \\
\text { ums. Pelveoperitonitis adhaesiva. Darm- } \\
\text { adhäsionen }\end{array}$ & $\begin{array}{l}\text { Geheilt entlassen. } \\
\text { 27. 6. } 1904\end{array}$ \\
\hline $\begin{array}{l}\text { Enucleation. Myektomie, } \\
\text { Ventrofixation. 23.2.1905 }\end{array}$ & $\begin{array}{l}\text { Gestieltes Adenomyom der hinteren Uterus- } \\
\text { wand, im hinteren Douglas gelegen. Kleine } \\
\text { multiple Fibroide des Fundus uteri }\end{array}$ & $\begin{array}{l}\text { Geheilt entlassen. } \\
\text { 21. 3. } 1905\end{array}$ \\
\hline $\begin{array}{l}\text { Vaginale Hysterektomie. } \\
\text { 15. 6. } 1905\end{array}$ & $\begin{array}{l}\text { Adenomyoma fundi uteri. Keine Ad- } \\
\text { häsionen }\end{array}$ & $\begin{array}{l}\text { Geheilt entlassen. } \\
\text { 2.7. } 1905\end{array}$ \\
\hline $\begin{array}{l}\text { Vaginale Hysterestomie. } \\
\text { 29.8. } 1905\end{array}$ & $\begin{array}{c}\text { Adenomyoma corporis uteri. Keine Ad- } \\
\text { häsionen }\end{array}$ & $\begin{array}{l}\text { Geheilt entlassen. } \\
22.9 .1905\end{array}$ \\
\hline $\begin{array}{l}\text { Vaginale Hysterektomie. } \\
\text { 29. 9. } 1905\end{array}$ & $\begin{array}{l}\text { Adenomyoma corporis uteri. Keine Ad- } \\
\text { häsionen }\end{array}$ & $\begin{array}{l}\text { Geheilt entlassen. } \\
\text { 19. 10. } 1905\end{array}$ \\
\hline $\begin{array}{l}\text { Abdominelle } \\
\text { pation. 9. 7. } 1906\end{array}$ & $\begin{array}{l}\text { Adenomyoma corporis uteri mit verkäsen- } \\
\text { der Tuberkulose. Geringe Adhäsionen }\end{array}$ & $\begin{array}{l}\text { Geheilt entlassen } \\
\text { 6. 8. } 1906\end{array}$ \\
\hline $\begin{array}{l}\text { Abdomin. Radicaloperation, } \\
\text { Appendektom. 10.10.1906 }\end{array}$ & $\begin{array}{l}\text { Tuboovarialeyste links, Adenomyoma cor- } \\
\text { poris uteri. Appendicitis, Periappendi- } \\
\text { citis adhaesiva. Keine Adhäsionen }\end{array}$ & $\begin{array}{l}\text { Geheilt entlassen } \\
\text { 24. 10. } 1906\end{array}$ \\
\hline $\begin{array}{l}\text { Amputatio supravagin. } \\
\begin{array}{l}\text { 6. 2. } 1907 . \quad \text { Exstirpatio } \\
\text { cervicis. 2.3. } 1907\end{array}\end{array}$ & $\begin{array}{l}\text { Adenocarcinoma uteri, Tuböovarialabscess } \\
\text { links, Salpingooophoritis rechts. Doppel- } \\
\text { seitige cystische Tubenwinkeladeno- } \\
\text { myome. Ausgedehnte Adhäsionen }\end{array}$ & $\begin{array}{l}\text { Geheilt entlasser } \\
\text { 30. 3. } 1907\end{array}$ \\
\hline $\begin{array}{l}\text { Amputatio supravaginalis m. } \\
\text { Entfernung der Adnexe. } \\
\text { 20.4. } 1907\end{array}$ & $\begin{array}{l}\text { Multiple Fibroide corporis uteri. Doppel- } \\
\text { seitige grosse Tubenwinkeladenomyome. } \\
\text { Hydrosalpinx sinistra cystis ovarii dextri, } \\
\text { Pelveoperitonitis adhaesiva }\end{array}$ & $\begin{array}{l}\text { Geheilt entlasser } \\
\text { 20.4. } 1907\end{array}$ \\
\hline
\end{tabular}

L. Landau's Monographie. 


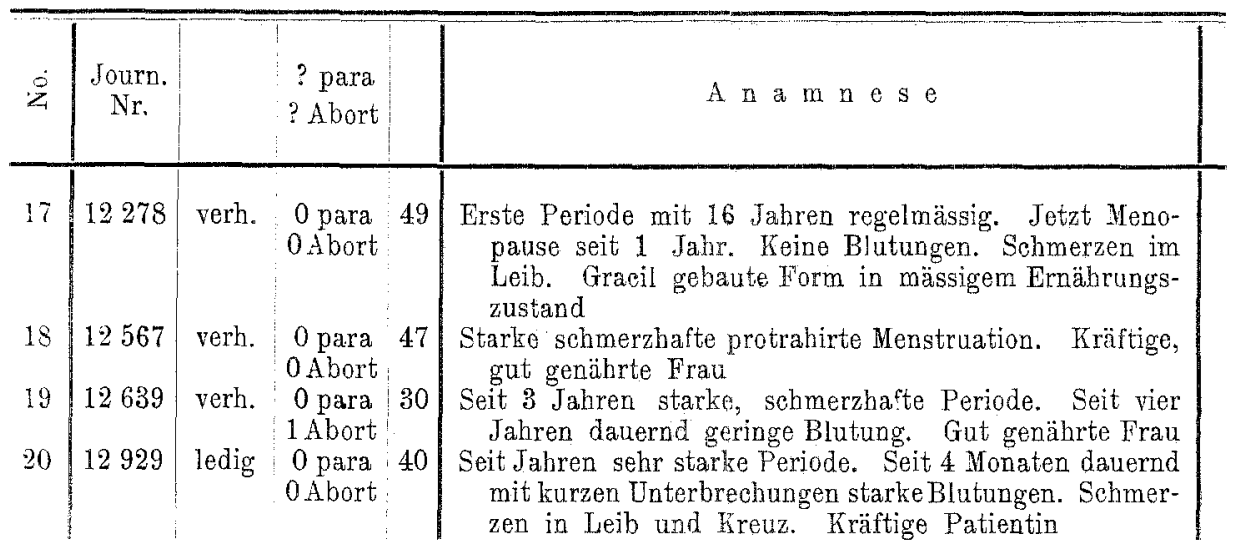

Zusammenfassend ergiebt sich Folgendes:

Von den 20 Patienten, deren Alter zwischen dem 32. und 51. Lebensjahre schwankt (Durchschnittsalter 41 Jahre), waren 2 ledig; von den 17 Verheiratheten waren 10 steril, 6 Patienten haben geboren $(4 \times 1$ para : $2 \times 3$ para). Es scheint, dass beim gewöhnlichen Fibromyom die Sterilität etwas geringer ist, immerhin aber ist die Beziehung des Adenomyoms zu Sterilität von nicht besonderer Bedeutung. Kudoh berechnet aus seiner Statistik $47 \mathrm{pCt}$. sterile Ehen bei Adenomyom gegenüber $20 \mathrm{pCt}$. bei Myom. Diese Zahl würde aber noch modificirt werden durch den Einschluss der in den letzten 2 Jahren veröffentlichten Fälle; so berichtete bejspielsweise Rutstein über 7 Adenomyomfälle aus der 0lshausen'schen Klinik, 6 Patientinnen waren verheirathet, davon nur Eine steril. Die Fertilität in den einzelnen Fällen war eine ganz beträchtliche ( 6 p., $6 \mathrm{Ab}$.; 5 p., $6 \mathrm{Ab}$.; 7 p., $0 \mathrm{Ab}$.).

Die Beschwerden, welche unsere Patientinnen zum Arzt führten, waren zumeist Menorrhagien und Metrorrhagien, unregelmässige Blutungen, die auch durch Abrasionen und Aetzungen keine Aenderungen erfabren haben. Dies betrifft von unseren 20 Patienten 11. 9 Patienten klagen über heftige Unterleibsschmerzen und Kreuzschmerzen, die z. Th. besonders heftig während der Menstruation auftraten; bei drei Frauen lokalisirten die Schmerzen besonders auf die Blasengegend und Störungen beim Urinlassen. Die Urinbeschwerden steigerten sich zweimal zu vollständiger Urinverhaltung, so dass diese Patientinnen des öfteren katheterisirt werden mussten. 


\begin{tabular}{|c|c|c|}
\hline operation & $B$ e $f u n d$ & \\
\hline $\begin{array}{l}\text { Amput. ut. supravaginalis. } \\
\text { 4. 7. } 1907\end{array}$ & $\begin{array}{l}\text { Adenomyoma corporis uteri. Pyosalpinx } \\
\text { duplex }\end{array}$ & $\begin{array}{l}\text { Geheilt entlassen. } \\
25.7 .1907\end{array}$ \\
\hline $\begin{array}{l}\text { Amput. ut. supravaginalis. } \\
\text { 14. 11. } 1907 \\
\text { Amput. ut. supravaginalis. } \\
\text { 30. 12. } 1907 \\
\text { Vaginale Hysterektomie. } \\
\text { 14. 5. } 1908\end{array}$ & $\begin{array}{l}\text { Adenomyoma corp. uteri. Peri. und Para- } \\
\text { metritis adhaesiva } \\
\text { Adenomyoma uteri. Graviditas tubaria } \\
\text { dextra } \\
\text { Adenomyoma uteri }\end{array}$ & $\begin{array}{l}\text { Geheilt entlassen. } \\
\text { 8. 12. } 1907 \\
\text { Geheilt entlassen. } \\
\text { 5. 1. } 1908 \\
\quad-\end{array}$ \\
\hline
\end{tabular}

Die Annahme W. A. Freund's, dass gewisse Zeichen von Infantilismus sich häufig bei Trägerinnen von Adenomyomen fänden, lässt sich durch unsere Fälle nicht stützen; von 10 unserer $\mathrm{Pa}$ tientinnen wird ausdrücklich berichtet, dass sie kräftig entwickelt waren. Auch die 1. Periode stellte sich, soweit dies erörtert wurde, nicht auffallend spät ein (13._16. Jahr).

In der Hälfte der Fälle waren neben dem Adenomyom entzündliche Veränderungen der Adnexe (Entzündung und entzündliche Tumorbildung) nachweisbar, ebenso Adhäsionsbildungen, z. Th. sehr ausgedehnte Verwachsungen mit Darm und Netz. Das Adenomyoma uteri ist, wie-schon v. Reklinghausen und und W. A. Freund hervorhoben, sehr häufig mit Pelveoperitonitis vergesellschaftet, jedenfalls häufiger, als dies beim Myom der Fall ist, wo sie Funke zu 10 pCt. der Fälle fand. Aber auch hierbei ist zu betonen, dass selbst bei grossen intraligamentären Adenomyomen chronisch adhäsive Peritonitis fehlen kann. Für die Diagnosenstellung ist dieses häufige Zusammentreffen von Adnextumoren und Adenomyom von nicht grosser Bedeutung, da man ja oft genug bei den gewöhnlichen Myomen schwere entzündliche Veränderungen der Adnexe sieht (Landau). Unter 233 in der Tübinger. Frauenklinik operirten Myomen hat Ballischwiller 43 mal Complicationen von Seiten der Adnexe festgestellt. Lauwers fand unter 200. Fällen $17 \mathrm{mal}$ Hämatome der Ovarien und $12 \mathrm{mal} \mathrm{Hydrosalpinx.} \mathrm{Nach} \mathrm{Meyer}$ lassen sich bei Myomen meist entzündliche Affectionen der Adnexe und zwar jeden Stadiums finden, welche z. Th. vorher 
bestanden haben, welche jedoch auch durch die Myome hervorgerufen oder doch verschlimmert sein können.

Immerhin aber sind in unseren Adenomyomfällen die schweren Adnexveränderungen sehr bemerkenswerth und klinisch deshalb wichtig, weil sie einerseits den Anlass zu starken Beschwerden gaben, andererseits aber die Operation selbst sehr complicirten. Es fanden sich in unseren Fällen alle möglichen Adnexveränderungen, wie Cystis ovarii, Follikelcyste des Ovariums, Tuboovarialcysten, Salpingitis, Hydrosalpinx, - Ovarialabscess - und Pyosalpinx.

Am Uterus selbst waren in den 20 Fällen neben dem Adenomyom $4 \mathrm{mal}$ multiple Fibroide vorhanden, je einmal war das Endometrium tuberculös und carcinomatös entartet, worauf später noch zurückzukommen ist.

Die Prognose der Erkrankung hält W. A. Freund für ernster als für das Myom gemeinhin. Es ist ohne Weiteres Freund zuzustimmen, dass jede medicamentöse Behandlung sowie auch kleinere operative Maassnahmen (Abrasio) keine endgültige Besserung, geschweige denn eine Heilung verursachen. Nur die Entfernung des erkrankten Uterus kommt in Retracht, sobald der Tumor solche Symptome macht, dass eine Operation indicirt ist (Landau). In unseren Fällen wurde 9 mal der vaginale Weg eingeschlagen (Hysterectomia vaginalis), $5 \mathrm{mal}$ wurde der Uterus durch supravaginale Amputation entfernt, 5 mal die abdominelle Radicaloperation vorgenommen, und in einem Fall, wo das Adenomyom mit breitem Stiel aus der hinteren Cteruswand vorsprang, genügte die Exstirpation dieses Tumors mit Erhaltung des Uterus selbst, nachdem aus demselben noch kleinere in Fundus gelegene Myome enucleirt waren. In allen Fällen wurde durch die Operation Heilung erzielt, die Mortalität betrug 0 pCt.

Die Diagnose wurde in keinem unserer 20 Fälle ante operationem anf Adenomyom gestellt. Entweder wurde der Befund als multiple Fibroide, oder als Myom mit Adnexentzündung gedeutet, oder aber es wurden die kleineren Adenomyome bei Combination mit Adnextumoren gänzlich übersehen, und die Diagnose wurde auf chronische Adnexerkrankung gestellt. In einigen Fällen wurde das Adenomyom als solehes erst während der Operation erkannt, wenn sich der Tumor ohne Kapselbildung erwies.

In der Mehrzahl der Fälle aber wurde die Diagnose auf 
Adenomyom erst durch die genaue pathologisch-anatomische Untersuchung der Präparate gestellt.

Interessant sind besonders einige Fälle, die vor der endgültigen Operation längere Zeit in klinischer Beobachtung standen, bei denen aber doch keine präsise Diagnose möglich war.

Der erste Fall (No. 7 der Tabelle) ist ausführlich in der Monographie L. Landau's publicirt (Journ.-No. 5899, 6146, Seite 17).

Bei der 35 jährigen Patientin war in der Annahme, dass es sich um ein Fibroid im hyperplastischen Uterus handle, dessen Enucleation beschlossen wurde, die vordere und hintere Kolpotomie gemacht worden. Die Tastung nach Eröffnung des Peritoneum ergab keinen localisirten Befund, der Uterus fühlte sich hart an, aber obne abgrenzbare Geschwulstbildung; es wurde deshalb von weiterem operativen Vorgehen Abstand genommen.

Die Schmerzen der Patientin liessen nicht nach, so dass nach einem halben Jahr die vaginale Radikaloperation vorgenommen wurde. An dem exstirpirten Uterus fand sich in der rechten Fundushälfte ein fast apfelgrosses typisches Adenomyom.

Landau bemerkt zu diesem Fall: „Wie soll der. Kliniker erschliessen, was ohne die Hülfe des Messers auch dem Anatomen verborgen bleiben muss."

Aehnlich sind auch die 3 anderen länger beobachteten Fälle gelagert.

Eine 30 jährige Frau (Fall 10 der Tabelle) war zum ersten Mal 1895 in die Klinik aufgenommen worden: es handelte sich damals um einen incarcerirten retroflectirten graviden Uterus; dieser wurde in Narkose aufgerichtet, und die Pat. verliess bald ohne Beschwerden die Klinik. 10 Jahre später suchte sie die Klinik wieder auf mit der Angabe, dass sie seit 2 Monaten fortgesetzt an Gebärmutterblutungen leide; die Periode war in den letzten 2 Jahren nicht mehr regelmässig wie früher. Pat. klagte ausserdem noch über Kopfschmerzen, Schwindel und Schmerzen in der linken Seite beim Stuhlgang.

Bei der kräftigen, im besten Ernährungszustand sich befindenden Frau wurde ein vergrösserter, derber, retroflectirter Uterus gefunden, deshalb nach Dilatation die Abrasio mucosae uteri vorgenommen. Die mikroskopische Untersuchung der Gewebsfetzen ergab Endometritis ohne Verdacht, so dass die Pat. nach 7 Tagen entlassen wurde.

Nachdem sich die Pat. nunmehr 5 Wochen lang wohl befunden hatte, trat ohne äussere Ursache wieder eine Blutung ein, die sich in den nächsten 14 Tagen sehr verschlimmerte.

Es wurde jetzt - der Befund hatte sich nicht geändert - die Hysterectomia vaginalis ausgeführt, die Exstirpation war nicht schwierig; da keine Adhäsionen vorhanden waren. Auf dem Durchschnitt des $11 \mathrm{~cm}$ langen Uterus fand sich in der Corpusmitte eine undeutliche Verdickung des Myometriums mit feinen rötlichen Oeffnungen im Muskelgewebe (mikroskopische Untersuchung: Adenomyom). 
Dioser Fall bietet eine typische Myomanamnese; wochenlange Blutungen und Schmerzen bei einer 40 jährigen Frau; die Blutungen kehren trotz ausgiebiger Abrasio und Aetzung des Cavum uteri wieder, so dass zur Exstirpation des verdickten (myomalösen?) Uterus geschritten werden musste. Erst die nachträgliche Untersuchung des Fundus uteri ergiebt als Ursache der Beschwerden das Adenomyom.

Eine 32 jährige Frau (No. 9 der Tabelle) kam wegen Sterilität und starken Fluors in Behandlung. Die Periode trat nur alle 6-8 Wochen, mit starken Kreuzschmerzen verbunden, auf. Die Untersuchung ergab einen vergrösserten. höckerigen, freibeweglichen Uterus und wurde vorerst in Narkose wegen des starken Ausflusses die Abrasio mit nachfolgender Aetzung vorgenommen.

Die Beschwerden nahmen im Laufe der nächsten Monate zu, ziehende Schmerzen im ganzen Leib, gelber Ausfluss, grosse Mattigkeit waren die Klagen. Nunmehr wurde neben dem höckerigen Uterus vom hinteren Scheidengewölbe aus ein klein apfelgrosser vom Uterus selbst nicht vollständig abgrenzbarer Tumor gefühlt, und es wurde die Enucleation dieses Tumors (Myom?) beschlossen.

Die Laparotomie zeigt, dass tief im Douglas retrocervical das kleinlaustgrosse Myom mit breitem Stiel aus der Hinterwand des Uterus herausgewachsen war. Die Entwicklung des Tumors gelang wegen der Tiefe des Sitzes nur mit grossen Schwierigkeiten; ausserdem wurden noch 3 kirschgrosse Myome aus der Vorderwand des Uterus enucleirt. Der retrocervical gelegene Tumor war ein Adenomyom.

Auch hier sprach nichts für das Vorhandensein eines Adenomyoms, da ja ausser dem breitbasigen Tumor der Hinterwand noch andere Myomknoten gefühlt worden waren und auch die Klagen der Patientin nichts Charakteristisches boten.

Besonders interessant ist endlich Fall 14 der Tabelle; hier hatte nicht einmal eine früher vorgenommene Laparotomie zur Diagnose geführt, obwohl anzunehmen ist, dass damals schon die Adenomyombildung im Uterus, wenn auch in geringerem Maasse vorhanden war.

Bei der 32 jährigen Pat. war am 9. 2. 03 eine faustgrosse rechtsseitige Tuboovarialcyste per laparotomiam entfernt worden. Damals wurde notirt: Lterus hart, gut beweglich. Am 9.5. 08 kam Pat. wieder in die Klinik mit der Angabe, dass seit der Operation die Periode immer unregelmässig gewesen sei, alle 3 Wochen auftrat, 7-8 Tage dauerte, und sich in der letzten Zeit unerträgliche Schmerzen beim Unwohlsein dazu gesellten.

Der Uterus wurde concentrisch vergrössert (mannsfaustgross) ohne Buckel gefühlt und links von demselben ein Caustgrosser cystischer Adnextumor, weshalb die abdominelle Radicaloperation (mit Exstirpation der Appendix) gemacht wurde. Das typische Adenomyom wurde erst durch den Anatomen beim Aufschneiden des vergrösserten Uterus gefunden. 
Es geht wohl aus den auszugsweise wiedergegebenen Krankenjournalen ohne Weiteres hervor, dass auch in diesen Fällen, die doch einer mehrfachen klinischen Beobachtung unterzogen worden waren, eine Abgrenzung der Diagnose rom Myom nicht möglich war.

\section{II.}

Von grossem klinischen und pathologisch-anatomischen Interesse ist die Frage, ob die Adenomyome in derselben Weise wie die Myome einer Degeneration anheimfallen.

Wir wissen, dass fast in jedem Myom älterer Frauen regressive Veränderungen, wie Nekrose, Verkalkung, Erweichung, Vereiterung zu finden sind. Diese degenerativen Zustände sind zum Theil klinisch durchaus nicht gleichgültiger Natur, sie können lebensgefährliche Zustände bedingen ${ }^{x}$ ).

Noch wichtiger ist die Gefahr der sarkomatösen Umwandlung und der Zusammenhang des Myoms mit dem Carcinoma corporis uteri. Die Zusammenstellung Olshausen's ergiebt unter 6470 Myomen 1,2 pCt. Sarkom und 4019 Myomen 2,1 pCt. Corpuscarcinom.

Beim Adenomyom liegen die Verhältnisse in Bezug auf die Degeneration wesentlich anders. Im Gegensatz zum Nyom sind sehr selten regressive Veränderungen nachgewiesen worden (L. Landau), nur ganz vereinzelt sind Blutungen, Pigmentablagerung und ganz geringe, circumscripte Verkalkung beschrieben (L. Pick). Vereiterung, Nekrose, Gangrän fehlen beim Adenomyom vollständig. $\mathrm{I}_{\text {. }}$ Landau erklärt dies mit dem Hinweise, dass durch die innige Verbindung des Adenomyoms mit dem Mutterboden in allen Theilen des Tumors günstige Ernährungsbedingungen vorhanden sind, und deshalb bei einer Enährungsstörung nur ein kleiner Tumorabschnitt leidet. Die geringen, bisher bekannten, regressiven Veränderungen sind fast nur auf den drüsigen Antheil beschränkt und

1) $\mathrm{Zu}$ diesen "benignen" Veränderungen ist besonders auch die Verkalkung des Myoms zu rechnen, die man als einen recht günstigen Ausgang anzusehen gewohnt ist. Auch in dor neuen Auflage des Veit'schen Handbuches sagt Olshausen, dass die Verkallung der Myome als Indication für. die Operation nicht in Betracht käme, die zahlreichen Publicationen französischer und englischer Autoren nicht berücksichtigend, die beweisen, dass gerade in spätesten Lebensalter das Myom nur wegen der eingetretenen Verkalkung, durch Druck auf die Blase oder auf den Darm bedrohliche Erscheinungen macht and zur Operation zwingt (siehe Grünbaum). 
beanspruchen kein weiteres klinisches Interesse. Nur ein Fall yon ungünstigem Ausgang eines grosseystisehen Adenomyoms ist bekannt (Dillmann), indem ein aussergewöhnlich grosses Cystadenom in's Jejunum perforirte und eine tödtliche Peritonitis verursachte.

Nicht so selten ist dagegen die Combination von Adenomyom und Carcinom beobachtet, die in 3 Gruppen geschieden werden kann:

1. Die maligne Degeneration des Adenomyoms selbst (primäre carcinomatöse Umwandlung des drüsigen Antheils).

2. Carcinom des Uterus mit gleichzeitiger carcinomatöser Degeneration des Adenomyoms.

3. Carcinom des Uterus und unabhängig davon Adenomyoma uteri.

Für die 3 Gruppen finden sich Beispiele aus der Literatur.

ad 1. Dillmann beschreibt 2 Fälle, bei denen sich Careinom primär in einem vorher bestehenden Adenomyom entwickelt hat mit ausgedehnter Metastasirung in Leber und im Peritoneum; in dem einen Fall fanden sich auch Metastasen im Darmtraktus und in den Lungen. Das Endometrium war in beiden Fällen frei von Carcinom. Aehnliche Befunde sind auch von Kaufmann, Rolly, Babescu veröffentlicht. Hierher ist vielleicht auch der merkwürdige Fall von Füth zu rechnen, in dem sich zwar das histologische Bild als nicht maligne erwies, das infiltrirende Wachsthum und das klinische Bild die Malignität rechtfertigt. Bei der 34 jährigen Patientin war die hintere Uteruswand adenomyomatös, die hintere Fläche entbehrte des serösen Ueberzuges, sah zernagt und zerfressen aus. Die Geschwulstmasse adhärirte fest mit der Rectumwand, und es gelang bei der Operation nicht, jm Gesunden zu arbeiten; die Uterusmucosa und die Schleimhaut der Cervix war etwas hyperplastisch, sonst intakt. Endlich wurde von Schwab ein Fall von multiplen carcinomatös entarteten Adenomyomen veröffentlicht, von denen ein hühnereigrosser Tumor im hinteren Douglas, kleinere kirschgrosse Myome am Fundus sassen. Dieselben wurden enucleirt (!) und die mikroskopische Untersuchung ergab die überraschende Diagnose, dass die in die Myome eingeschlossenen Drüsen carcinomatös waren; auch hier war die Mucosa uteri intakt.

ad 2. Combination von Carcinom des Uterus mit Carcinom des Adenomyoms ist von v. Recklinghausen beschrieben, der annimmt, dass der von der Uterusmucosa her eindringende Krebs 
die präexistirenden benignen Adenomyome ergriff; ebenso sind die Fälle Cullen's aufufassen, auch in 2 Fällen R. Meyer's war der unmittelbare Zusammenhang des carcinomatösen Adenomyoms mit der carcinomatösen Mucosa uteri klar. Babes demonstrirte ein fortgeschrittenes Cervixcarcinom (Cancroid), im Fundus uteri sass ein Adenomyom mit einzelnen Drüsen, dessen Epithelien eine starke Mehrschichtung und Vordringen in's umliegende Gewebe zeigten.

ad 3. Zu der 3. Gruppe, zu der die Fälle gerechnet werden, in denen sich neben dem Adenomyom in irgend einem Abschnitt des Uterus ein Carcinom findet (v. Recklinghausen, Cullen u. a), gehört auch ein Fall unserer Beobachtung, dessen Krankengeschichte ich etwas ausführlicher wiedergeben will, da dieselbe auch von anderen Gesichtspunkten aus ein klinisches Interesse bietet.

Die 50 jährige Patientin (Fall 15 der Tabelle), welche niemals geboren hatte, gab an, vor 7 Jahren wegen einer Unterleibserkrankung in Behandlung gewesen zu sein; es wurde damals wegen gelben Ausflusses eine Ausschabung der Gebärmutter vorgenommen.

Sie fühlte sich bis vor 5 Monaten ganz gesund, damals bekam sie Urinbeschwerden; sie konnte zeitweise keinen Urin lassen, so dass sie vom Arzte katheterisirt werden musste; es wurde eime Unterleibsgeschwulst constatirt, und der Patientin die Operation angerathen, die sie aber bis jetzt verweigerte. Jetzt kommt Pat. wegen Urinbeschwerden, und Schmerzen an den äusseren Geschlechtsteilen, wo sich ein "Geschwür" gebildet habe.

Es bestehen sonst keine Unterleibsschmerzen oder Kreuzschmerzen, Appetit und Schlaf ist gut, Stuhlgang regelmässig.

Die Menstruation war immer regelmässig alle 4 Wochen, mit Ausnahme der letzten 2 Jahre, wo sie monatelang aussetzte: letzte Regel vor 4 Wochen, vorletzte Menses vor 3 Monaten.

Die geringen Beschwerden stehen in keinem Verhältnis zu dem ausserordentlich schweren Krankheitsbild, das sich bei der Operation fand. (Oper. 6. 2. 07.)

Nach Eröffnung der Peritoneums stellte sich hinter dem sehr vergrösserten Uterus ein kindskopfgrosser, in Adhaesionen eingebetteter Tumor ein, welcher beim Versuch der Lösung platzte und eine rothbraune, zum Theil eitrige Flüssigkeit entleerte (Tuboovarialabscess links). Die Tumorwand war mit der Hinterwand des Uterus und mit dem Rectum ausgedehnt verwachsen und konnte nur mit grosser Mühe losgelöst werden. Nunmehr wurde der mannsfaustgrosse hauptsächlich in die Breite gehende Uterus mobilisirt, durch supravaginale Amputation entfernt. Der zurückgelassene Cervixstumpf wurde mit Peritoneum überkleidet und dann nach ausgiebiger Drainage des Tumorbettes, aus dem es ganz in der Tiefe parenchymatös blutete, die Bauchhöhle geschlossen.

Der Uterus bietet schon makroskopisch (siehe Figur) das typische Bild eines Adenomyoms, - er ist stark verbreitert, auf der Oberfläche der rechten Vorderhälfte prominiren zahlreiche transparente Cysten und und Cystchen, die auch auf den Durchschnitten der Tumoren von Steck- 
nadelknoptgrösse bis über Wallnussgrösse sich präsentiren. Die Cysten sind mit einer serösen. Flïssigkeit von röthlichem Schimmer angefüllt, and von Musculatur umscheidet. Die Adenomyome nehmen zum grössten Theil die beiden Uterushörner ein und reichen bis tief in die Corpusmasse hinein.

Weder der Befund, noch die Anamnese deuteten im Entferntesten auf ein Carcinom hin, dieses wurde erst beim Einschneiden der ca. $2 \mathrm{~cm}$ dicken Corpusvorderwand bis in die Mucosa hinein entdeckt. Nach Eröffnung quollen aus dem Corpus uteri weiche grauröthliche Massen hervor, die auch im Fundus uteri sassen. Die mikroskopische Untersuchung ergab ein charakteristisches Adenocarcinom, das den grössten Theil der Mucosa ergriffen hatte. Die Adenomyome selbst, von denen verschiedene Stückchen mikroskopisch untersucht wurden,

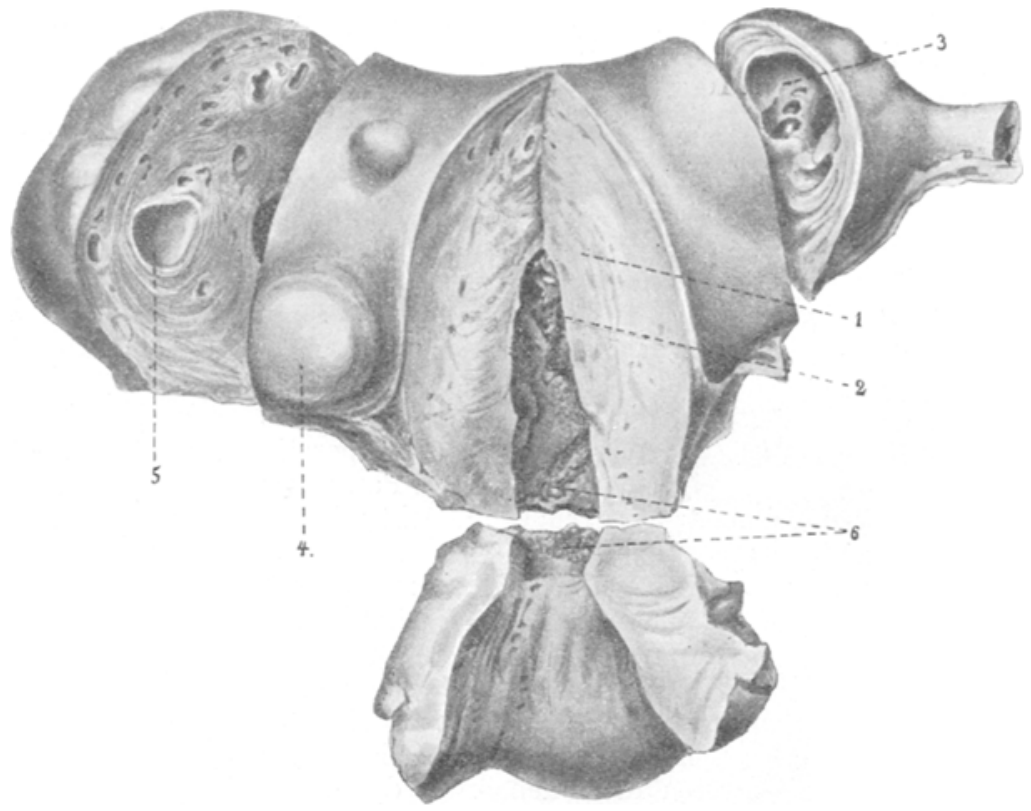

Adenomyoma corporis uteri mit Idenocarcinoma uteri.

1. Myometrium (Vorderwand des Uterus). 2. Adenocarcinoma endometrii. 3. Tubenwinkeladenomyom. 4. Subseröse Adenocyste. 5. Cystenbildung im Adenomyom. 6. Adenocarcinom der Cervixschnittgrenze.

waren frei ron Carcinom; auch die beiden Tuben zeigten nur das Bild chronischer Entzündung, die Mucosa der rechten Tube war mächtig gewuchert, enthielt Rundzellenanhäufungen, aber keine Carcinomnester. Dagegen fanden sich an der Cervixsehnittgrenze Carcinomnester, so dass mit Sicherheit angenommen werden musste, dass auch in dem zurückgelassenen Cervixstumpf Carcinom sich finden würde.

Es musste deshalb, wenn nicht die ganze Operation werthlos sein sollte, nachträglich noch der Cervixstumpf exstirpirt werden. Dies geschah auch, nachdem sich die Patientin von der ersten Operation gut erholt hatte, $3 \frac{1}{2}$ Wochen später. 
Die Portio wurde von der Vagina her gefasst, umschnitten, hierauf die Blase abgelöst, was keine Schwierigkeiten machte. Dem Operateur gelang es dann, vom hinteren Douglas aus über den Cervixstumpf hinüberzukommen und denselben zu mobilisiren. Nach Unterbindung der seitlichen Gefässe und Anlegen von Dauerklemmen, welche Peritoneum und Scheide zugleich fassen, wurde die Cervix abgeschnitten. Dieselbe war mit Peritoneum überkleidet, welches eine glatte Nahtnarbe zeigte1). In den obersten Cervixabschnitt des Stumpfes fanden sich, der Annahme gemäss, kleine Carcinomnester.

Die Patientin überstand auch diesen Eingriff gut, nur bildete sich eine kleine Blasenscheidenfistel, die bei der Entlassung nach 4 Wochen noch nicht ganz geschlossen war. Pat. war $1 / 2$ Jahr später, als sie sich einer Nachuntersuchung unterzog, frei von Recidiv.

Bemerkenswerth an unserem Fall ist ausser dem gleichzeitigen Vorkommen von Adenomyom und Adenocarcinom in demselben Uterus, die gänzliche Symptomlosigkeit des sehr ausgebreiteten Carcinoms. Die intelligente Patientin machte ganz bestimmte Angaben über ihre Menstruationsverhältnisse, die dem Typus der Menstruation einer im Klimacterium sich befindlichen Frau entsprachen. Die Symptome, welche die Patientin veranlassten, zum Arzt zu gehen, waren einzig und allein, Blasenbeschwerden, welche durch den Druck des adenomyomatösen Uterus hinreichend motivirt waren, umsomehr, als der hinter dem Uterus gelegene, den Douglas einnehmende kindskopfgrosse Tuboovarialabscess den Uterus nach vorn drängte.

Nach R. Meyer ist das gleichzeitige Vorkommen von Adenomyom und Carcinom im Uterus nicht so häufig als Carcinombefund beim myomatösen Uterus überhaupt, es scheint aber doch, nach dem nicht so spärlichen casuistischen Material schliessend, die Annahme berechtigt zu sein, auch nach dieser Richtung hin eine Congruenz mit dem Myoma uteri zu verzeichnen. Vielleicht ist durch den drüsigen Antheil des Adenomyoms der Boden für eine carcinomatöse Degeneration eher gegeben als beim Myom selbst. Sarkomatöse Entartung des Adenomyoms dagegen ist bisher nur in ganz wenigen Fällen bekannt (Kaufmann, Iwanoff, Bauereisen).

Zum Schlusse noch ist die tuberculöse Degeneration des Adenomyoms zu erwähnen, welche bis jetzt 5 mal beobachtet wurde,

1) In dem Cerrixgewebe fanden sich noch gut erhaltene, farblos gewordene, Jodcatgutfäden, die bei der ersten $-31 / 2$ Wochen zurückliegenden - Operation gelegt worden waren, ein Zeichen für die Widerstandskraft, die das Jodcatgut der Resorption entgegensetzt. 
darunter 1 Fall unserer Reihe (Fall 13 der Tabelle), der zum Gegenstand einer eignen Untersuchung gemacht wurde (Grünbaum) ${ }^{1}$ ). Bei der 45 jährigen Patientin war seit 5 Jahren ein Myom constatirt worden, das durch Verwachsungen mit der Blase schliesslich solche Beschwerden verursachte, dass zur Operation geschritten werden musste. Die Untersuchung des durch abdominelle Totalexstirpation gewonnenen Cterus ergab, dass es sich um ein diffuses. Adenomyoma corporis uteri mit verkäsender Tuberculose handelte. Die Verkäsung hielt sich hauptsächlich an den adenomatösen Parthien des Tumors und hatte in den periphersten Schichten zu ausgedehnter Nekrose geführt. In Schnittpräparaten wurden Tuberkelbacillen gefunden.

Da die Tuberculose in einem deutlichen Zusammenhang zu den adenomatösen Wucherungen selbst steht, liegt es nahe, anzunehmen, dass die Tubereulose als Aetiologie für die Entstehung der Adenomyome angesehen werden könne. Dies trifft aber für die bisher bekannten Fälle nicht zu, sondern die adenomyomatösen Wucherungen begünstigen als Locus minoris resistentiae das Eindringen der Tuberculose vom Endometrium her. Auch in unserem Fall wurde angenommen, dass von einem alten Herd in der Lunge auf dem Blutwege das Endometrium secundär inficirt wurde, und von. da aus die Tuberculose in das Adenomyom weiter vordrang.

Fasse ich kurz die Ergebnisse vorliegender Untersuchung zusammen, so ergiebt sich eine volle Uebereinstimmung mit den Ansiehten von L. Landau, Kudoh-Polano, dahingehend, dass das Adenomyom kein specifisches Krankheitsbild darbietet, sich vielmehr in Uebereinstimmung mit dem klinischen Bild des Myoms befindet. Nur in den seltensten Fällen dürfte es gelingen, die Diagnose auf Adenomyom ante operationem zu stellen.

Das Adenomyom ist im Vergleich zum Myome häufiger mit. Pelveoperitonitis und Adnexveränderungen vergesellschaftet.

Schwere degenerative Processe, wie Vereiterung, Verjauchung, Gangrän treten beim Adenomyom nicht auf, dagegen ist carcinomatöse, sarkomatöse und tuberculöse Entartung des Adenomyoms des öfteren beobachtet.

1) Zu den Fällen von v. Recklinghausen, Lichtenstein und Hösli, welche ausführlich in der erwähnten Arbeit wiedergegeben sind, tritt ein neuer Fall ron Archambauld et Pearce hinzu, der sich klinisch und anatomisch enge an den Fall der Landau'schen Klinik anschliesst. 


\section{Li t e r a t ur.}

Archambauld et Pearce, Adenomyome de l'utérus avec tuberculose. Arch. de Gynaec. (Pozzi.) 1907.

Babes, Demonstration. Berliner gynäk. Gesellsch. 1906. Ref. Zeitschr. f. Gyn. u. Geburtsh. 1906.

Ballisehwiller, s. Döderlein-Krönig, Operat. Gynäk, 1905. S. 372. Callen, Adenomyome des Uterus. Berlin 1903.

Dillmann, Adenomyome des Uterus und ibre Beziehung zum Krebs. Zeitschr. für Krebsforschung. 1904. Bd. 2.

W. A. Freund, s. v. Recklinghausen.

Funk, Beiträge zur klinischen Diagnostik . . . . Deutsche med. Wochenschr. 1903. No. 49.

Füth, Beitrag zur Kasuistik der Adenomyome des Uterus. Centralbl. f. Gyn. 1903. S. 629 .

Grünbaum, Klinischer Beitrag zur Verkalknng des Uteruskörpers. Dieses Archiv. Bd. 80.

Grünbaum, Adenomyoma corporis uteri mitTuberculose. Dieses Arch. Bd. 81 . I wa n off, Drüs. cystisches Uterusfibrom, complicirt durch Sarkom and Carcinom. Monatsschr. f. Gyn. u. Geb. 1898. Bd. 7.

Kaufmann, Lehrbuch der path. Anatomie.

$\mathrm{Kudoh}$, Klinische und anatomische Beiträge zur Adenomyombildung im Uteruskörper. Inaug.-Diss. Würzburg 1905.

L. Landau, Anatomische und klinische Beiträge zur Lehre von den Myomen am weiblichen Sexualapparat. Berlin 1899.

L. Landau, Symptomatologie und Behandlung der Myome. Deutsche Klinik.

Lauwers, Annal. de Gyn. 1895. Cit. naoh Olshausen.

R. Meyer, Die Myome und Fihrome des Uterus. Veit's Handbuch der Gynäk. II. Autl. Bd. I.

Olshausen, Die abdominellen Myomoperationen. Veit's Handbuch. Bd. I. II. Auflage.

L. Pick, Ein neuer Typus des voluminösen paroovoralen Adenomyoms. Dieses Archiv. Bd. 54.

Polan 0, Das klinische Verhalten des Adenomyoma uteri. Zeitschr. f. Geburtsh. u. Gyn. Bd. 54. H. 2.

v. Recklinghansen, Die Adenome und Cystadenome des Uterus und der Tubenwandung. 1896.

Rolly, Adenomyom mit Uebergang in Carcinom. Virchow's Archiv. Bd. 150.

v. Rosthorn, Die klinische Diagnose der Adenomyome. Medicinische Klinik. No. 9. 1905.

Schwab, Multiple Adenomyomata uteri in carcinomatöser Degeneration. Beitr. zur Geburtsh. u. Gyn. Bd. 12. 1907. 